\title{
Electronic Art concerning the Sony's Performances in Businesses
}

\author{
Gabriela OPAIT ${ }^{\star}$
}

\begin{tabular}{l}
\hline \multicolumn{1}{c}{ A R T I C L E I N F O } \\
\hline Article history: \\
Accepted March 2021 \\
Available online May 2021 \\
\hline JEL Classification \\
C1, C12, C2 \\
Keywords: \\
Sony Electronics, Sony Pictures \\
Entertainment, Sony Music \\
Entertainment \\
\hline
\end{tabular}

\begin{abstract}
A B S T R A C T
The Sony Corporation exhibits a magnificent brand which fascinates through a lot of electronics products and a marvellous quality concerning the music and motion picture which reached out to the loving public. In the "electronic land", the Sony Corporation shines through the performances of the televisions with android, computers, telecomunication and audio-video devices. The aim of this original research consists in to show through the forecasts the excellency in businesses as effect of the strategic management which "chairs" in the Sony Corporation.
\end{abstract}

(C) 2021 EAI. All rights reserved.

\section{Introduction}

The Sony Corporation's establishment was achived in 1946, by Akio Morita and Masaru Ibuka, in the "flowers of cherry's country”, namely Japan, with the headquarters in Tokyo. From the start, this japanese conglomerate was a real treasure of inventions, where we can identify: the Walkman, the first radio transistor, the first mobile CD player, the first cassette with magnetic tape and the Playstation game console. In the first segment of this original article, we can see the protocol which must be applied for to achieve the worldwide revenues's forecast between 2021-2025, concerning the Sony Corporation. In the second episode of this research, we can observe the algorithm used for to project the worldwide revenues's prognosis, in the same period 2021-2025, regarding the Sony's Imaging Product\&Solutions. In the third segment of this article, we can remark the technique applied for to sculpt the forecasts between 2021-2025, concerning the worldwide revenues for Sony's Home Entertainment and Sound Products\&Solutions. In the fourth episode of this paper, we can make out the tactics through we can build the worldwide revenues's forecast concerning the Sony's Music Entertainment, between 2021-2025. The prediction's method was applied in this article for to display the excellent performances achieved by the Sony Corporation in the businesses's world. The past master who shaped the constitution of the „Least Squares Method”, which is the „best ingredient” for to pattern previsions, was Johann Carl Friedrich Gauss.

2. The survey concerning the worldwide revenues for the Sony Corporation, in the period 2007-2020, which has as target them estimation between 2021-2025

Table 1. The Sony Corporation's worldwide revenues, between 2007-2020

\begin{tabular}{|c|c|}
\hline YEARS & $\begin{array}{c}\text { THE SONY CORPORATION'S } \\
\text { WORLDWIDE REVENUES } \\
\text { (billions \$) } \\
\left(\xi_{i}\right)\end{array}$ \\
\hline 2007 & $\mathbf{8 8 , 7 1}$ \\
\hline 2008 & 78,88 \\
\hline 2009 & 77,57 \\
\hline 2010 & $\mathbf{8 6 , 5 2}$ \\
\hline 2011 & 79,19 \\
\hline 2012 & 72,35 \\
\hline 2013 & 75,41 \\
\hline 2014 & 68,47 \\
\hline 2015 & 71,73 \\
\hline 2016 & 67,89 \\
\hline 2017 & 77,04 \\
\hline
\end{tabular}




\begin{tabular}{|c|c|}
\hline \multirow{2}{*}{ YEARS } & $\begin{array}{c}\text { THE SONY CORPORATION'S } \\
\text { WORLDWIDE REVENUES } \\
\text { (billions \$) } \\
\left(\xi_{i}\right)\end{array}$ \\
\hline $\mathbf{2 0 1 8}$ & $\mathbf{7 8 , 1 4}$ \\
\hline $\mathbf{2 0 1 9}$ & $\mathbf{7 6 , 9 2}$ \\
\hline $\mathbf{2 0 2 0}$ & $\mathbf{7 5 , 9 9}$ \\
\hline
\end{tabular}

- if the proceeding's structure, which draws up the trend for the $\xi$ variable, where $\xi=$ the Sony Corporation's worldwide revenues, selects a linear exposure, $\xi_{t_{i}}=a+b \cdot t_{i}, a$ and $b$ will be [2]:

Table 2. The „esplanade” regarding the Sony Corporation's worldwide revenues, if this brings forward a linear "rally"

\begin{tabular}{|c|c|c|c|c|c|c|}
\hline \multirow{2}{*}{ YEARS } & \multirow{2}{*}{$\begin{array}{c}\text { THE SONY } \\
\text { CORPORATION'S } \\
\text { WORLDWIDE } \\
\text { REVENUES } \\
\text { (billions \$) } \\
\left(\xi_{i}\right)\end{array}$} & \multicolumn{5}{|c|}{ LINEAR TENDENCY } \\
\hline & & $t_{i}$ & $t_{i}^{2}$ & $t_{i} \xi_{i}$ & $\xi_{t_{i}}=a+b t_{i}$ & $\left|\xi_{i}-\xi_{t_{i}}\right|$ \\
\hline 2007 & 88,71 & -7 & 49 & $-620,97$ & 80,99589286 & 7,71 \\
\hline 2008 & 78,88 & -6 & 36 & $-473,28$ & 80,39250000 & 1,51 \\
\hline 2009 & 77,57 & -5 & 25 & $-378,85$ & 79,78910715 & 2,22 \\
\hline 2010 & 86,52 & -4 & 16 & $-346,08$ & 79,18571429 & 7,33 \\
\hline 2011 & 79,19 & -3 & 9 & $-237,57$ & 78,58232143 & 0,61 \\
\hline 2012 & 72,35 & -2 & 4 & $-144,70$ & 77,97892857 & 5,63 \\
\hline 2013 & 75,41 & -1 & 1 & $-75,41$ & 77,37553572 & 1,97 \\
\hline 2014 & 68,47 & +1 & 1 & $+68,47$ & 76,16875000 & 7,70 \\
\hline 2015 & 71,73 & +2 & 4 & $+143,46$ & 75,56535715 & 3,84 \\
\hline 2016 & 67,89 & +3 & 9 & $+203,67$ & 74,96196429 & 7,07 \\
\hline 2017 & 77,04 & +4 & 16 & $+308,16$ & 74,35857143 & 2,68 \\
\hline 2018 & 78,14 & +5 & 25 & $+390,70$ & 73,75517858 & 4,38 \\
\hline 2019 & 76,92 & +6 & 36 & $+461,52$ & 73,15178572 & 3,77 \\
\hline 2020 & 75,99 & +7 & 49 & $+531,93$ & 72,54839286 & 3,44 \\
\hline TOTAL & 1074,81 & 0 & 280 & $-168,95$ & & 59,86 \\
\hline
\end{tabular}

$$
\begin{gathered}
a=\frac{\sum_{i=1}^{n} \xi_{i} \sum_{i=1}^{n} t_{i}{ }^{2}-\sum_{i=1}^{n} \xi_{i} t_{i} \sum_{i=1}^{n} t_{i}}{n \sum_{i=1}^{n} t_{i}{ }^{2}-\left(\sum_{i=1}^{n} t_{i}\right)^{2}}=\frac{1074,81 \cdot 280}{14 \cdot 280}=76,77214286 b=\frac{n \sum_{i=1}^{n} \xi_{i} t_{i}-\sum_{i=1}^{n} t_{i} \sum_{i=1}^{n} \xi_{i}}{n \sum_{i=1}^{n} t_{i}{ }^{2}-\left(\sum_{i=1}^{n} t_{i}\right)^{2}}=\frac{14 \cdot(-168,95)}{14 \cdot 280}=-0,603392857 \\
v_{I}=\left[\frac{\sum_{i=1}^{m}\left|\xi_{i}-\xi_{t_{i}}\right|}{n}: \frac{\sum_{i=1}^{m} \xi_{i}}{n}\right] \cdot 100=\frac{\sum_{i=1}^{m}\left|\xi_{i}-\xi_{t_{i}}^{I}\right|}{\sum_{i=1}^{m} \xi_{i}} \cdot 100=\frac{59,86}{1074,81} \cdot 100=5,57 \%
\end{gathered}
$$

- if the proceeding's structure, which draws up the trend for $\xi$ variable, where $\xi=$ the Sony Corporation's worldwide revenues, selects a parabolic exposure, $\xi_{t_{i}}=a+b \cdot t_{i}+c t_{i}^{2}, a$ and $b$ will be [2]:

\begin{tabular}{|c|c|c|c|c|c|c|c|}
\hline \multirow{2}{*}{ YEARS } & \multirow{2}{*}{$\begin{array}{c}\text { THE SONY } \\
\text { CORPORATION'S } \\
\text { WORLDWIDE } \\
\text { REVENUES } \\
\text { (billions \$) } \\
\left(\xi_{i}\right)\end{array}$} & \multicolumn{6}{|c|}{ PARABOLIC TENDENCY } \\
\hline & & $t_{i}$ & $t_{i}^{2}$ & $t_{i}^{4}$ & $t_{i}^{2} \xi_{i}$ & $\xi_{t_{i}}=a+b t_{i}+c t_{i}^{2}$ & $\mid \xi_{i}-\xi_{t_{i}}$ \\
\hline 2007 & 88,71 & -7 & 49 & 2401 & 4346,79 & 86,68868866 & 2,02 \\
\hline 2008 & 78,88 & -6 & 36 & 1296 & 2839,68 & 83,53335285 & 4,65 \\
\hline 2009 & 77,57 & -5 & 25 & 625 & 1939,25 & 80,77062365 & 3,20 \\
\hline 2010 & 86,52 & -4 & 16 & 256 & 1384,32 & 78,40050105 & 8,12 \\
\hline 2011 & 79,19 & -3 & 9 & 81 & 712,71 & 76,42298507 & 2,77 \\
\hline 2012 & 72,35 & -2 & 4 & 16 & 289,40 & 74,83807569 & 2,49 \\
\hline 2013 & 75,41 & -1 & 1 & 1 & 75,41 & 73,64577292 & 1,76 \\
\hline 2014 & 68,47 & +1 & 1 & 1 & 68,47 & 72,43898721 & 3,97 \\
\hline
\end{tabular}

Table 3. The „esplanade” concerning the Sony Corporation's worldwide revenues, if this brings forward a quadratic „rally” 


\begin{tabular}{|c|c|c|c|c|c|c|c|}
\hline \multirow{2}{*}{ YEARS } & \multirow{2}{*}{$\begin{array}{c}\text { THE SONY } \\
\text { CORPORATION'S } \\
\text { WORLDWIDE } \\
\text { REVENUES } \\
\text { (billions \$) } \\
\left(\xi_{i}\right)\end{array}$} & \multicolumn{6}{|c|}{ PARABOLIC TENDENCY } \\
\hline & & $t_{i}$ & $t_{i}^{2}$ & $t_{i}^{4}$ & $t_{i}^{2} \xi_{i}$ & $\xi_{t_{i}}=a+b t_{i}+c t_{i}^{2}$ & $\mid \xi_{i}-\xi_{t_{i}}$ \\
\hline 2015 & $\mathbf{7 1 , 7 3}$ & +2 & 4 & 16 & 286,92 & 72,42450426 & 0,69 \\
\hline 2016 & 67,89 & +3 & 9 & 81 & 611,01 & 72,80262793 & 4,91 \\
\hline 2017 & 77,04 & +4 & 16 & 256 & 1232,64 & 73,57335820 & 3,47 \\
\hline 2018 & 78,14 & +5 & 25 & 625 & 1953,50 & 74,73669508 & 3,40 \\
\hline 2019 & 76,92 & +6 & 36 & 1296 & 2769,12 & 76,29263856 & 0,63 \\
\hline 2020 & 75,99 & +7 & 49 & 2401 & 3723,51 & 78,24118866 & 2,25 \\
\hline TOTAL & 1074,81 & 0 & 280 & 9352 & 22232,73 & & 44,33 \\
\hline
\end{tabular}

$$
\begin{gathered}
a=\frac{\sum_{i=1}^{n} t_{i}^{4} \sum_{i=1}^{n} \xi_{i}-\sum_{i=1}^{n} t_{i}^{2} \sum_{i=1}^{n} t_{i}^{2} \cdot \xi_{i}}{n \sum_{i=1}^{n} t_{i}{ }^{4}-\left(\sum_{i=1}^{n} t_{i}^{2}\right)^{2}}=\frac{9352 \cdot 1074,81-280 \cdot 22232,73}{14 \cdot 9352-280^{2}}=72,84607676 \\
b=\frac{\sum_{i=1}^{n} \xi_{i} t_{i}}{\sum_{i=1}^{n} t_{i}^{2}}=-\frac{168,95}{280}=-0,603392857 \\
c=\frac{n \cdot \sum_{i=1}^{n} t_{i}^{2} \cdot \xi_{i}-\sum_{i=1}^{n} t_{i}^{2} \cdot \sum_{i=1}^{n} \xi_{i}}{n \sum_{i=1}^{n} t_{i}{ }^{4}-\left(\sum_{i=1}^{n} t_{i}^{2}\right)^{2}}=\frac{14 \cdot 22232,73-280 \cdot 1074,81}{14 \cdot 9352-280^{2}}=0,196303304 \\
v_{I I}=\left[\frac{\sum_{i=1}^{m} \mid \xi_{i}-\xi_{t_{i}}{ }^{I I}}{n}: \frac{\sum_{i=1}^{m} \xi_{i}}{n}\right] \cdot 100=\frac{\sum_{i=1}^{m}\left|\xi_{i}-\xi_{t_{i}}\right|}{\sum_{i=1}^{m} \xi_{i}} \cdot 100=\frac{44,33}{1074,81} \cdot 100=4,12 \%
\end{gathered}
$$

- if the proceeding's structure, which draws up the trend for $\xi$ variable, where $\xi=$ the Sony Corporation's worldwide revenues, selects an exponential exposure, $\xi_{t_{i}}=a b^{t_{i}}, a$ and $b$ will be [2]:

\begin{tabular}{|c|c|c|c|c|c|c|c|}
\hline \multirow{2}{*}{ YEARS } & \multirow{2}{*}{$\begin{array}{l}\text { THE SONY } \\
\text { CORPORATION'S } \\
\text { WORLDWIDE } \\
\text { REVENUES } \\
\text { (billions\$) } \\
\left(\xi_{i}\right)\end{array}$} & \multicolumn{6}{|c|}{ EXPONENTIAL TENDENCY } \\
\hline & & $t_{i}$ & $\lg \xi_{i}$ & $t_{i} \lg \xi_{i}$ & $\lg \xi_{t_{i}}=\lg a+t_{i} \lg b$ & $\xi_{t_{i}}=a b^{t_{i}}$ & $\left|\xi_{i}-\xi_{t_{i}}\right|$ \\
\hline 2007 & 88,71 & -7 & 1,947972579 & $-13,63580805$ & 1,908343049 & 80,97352558 & 7,74 \\
\hline 2008 & 78,88 & -6 & 1,896966902 & $-11,38180141$ & 1,904872403 & 80,32900785 & 1,45 \\
\hline 2009 & 77,57 & -5 & 1,889693791 & $-9,448468957$ & 1,901401764 & 79,68962149 & 2,12 \\
\hline 2010 & 86,52 & -4 & 1,937116511 & $-7,748466043$ & 1,897931125 & 79,05532438 & 7,46 \\
\hline 2011 & 79,19 & -3 & 1,898670343 & $-5,696011029$ & 1,894460486 & 78,42607602 & 0,76 \\
\hline 2012 & 72,35 & -2 & 1,859438535 & $-3,718877071$ & 1,890989847 & 77,80183623 & 5,45 \\
\hline 2013 & 75,41 & -1 & 1,877428941 & $-1,877428941$ & 1,887519208 & 77,18256512 & 1,77 \\
\hline 2014 & 68,47 & +1 & 1,835500328 & $+1,835500328$ & 1,880577930 & 75,95877112 & 7,49 \\
\hline
\end{tabular}

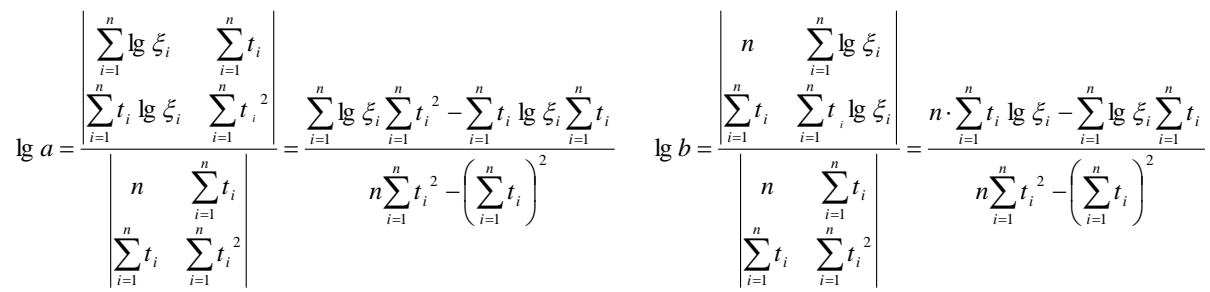

Table 4. The „esplanade” concerning the Sony Corporation's worldwide revenues, if this brings forward an exponential "rally" 


\begin{tabular}{|c|c|c|c|c|c|c|c|}
\hline \multirow{2}{*}{ YEARS } & \multirow{2}{*}{$\begin{array}{c}\text { THE SONY } \\
\text { CORPORATION'S } \\
\text { WORLDWIDE } \\
\text { REVENUES } \\
\text { (billions\$) } \\
\left(\xi_{i}\right)\end{array}$} & \multicolumn{6}{|c|}{ EXPONENTIAL TENDENCY } \\
\hline & & $t_{i}$ & $\lg \xi_{i}$ & $t_{i} \lg \xi_{i}$ & $\lg \xi_{t_{i}}=\lg a+t_{i} \lg b$ & $\xi_{t_{i}}=a b^{t_{i}}$ & $\left|\xi_{i}-\xi_{t_{i}}\right|$ \\
\hline 2015 & 71,73 & +2 & 1,855700831 & $+3,711401662$ & 1,877107291 & 75,35417007 & 3,62 \\
\hline 2016 & 67,89 & +3 & 1,831805809 & $+5,495417426$ & 1,873636652 & 74,75438140 & 6,86 \\
\hline 2017 & 77,04 & +4 & 1,886716274 & $+7,546865096$ & 1,897931125 & 79,05532438 & 2,02 \\
\hline 2018 & 78,14 & +5 & 1,892873407 & $+9,464367034$ & 1,866695374 & 73,56908828 & 4,57 \\
\hline 2019 & 76,92 & +6 & 1,886039276 & $+11,31623565$ & 1,863224735 & 72,98350814 & 3,94 \\
\hline 2020 & 75,99 & +7 & 1,880756445 & $+13,16529511$ & 1,859754096 & 72,40258898 & 3,59 \\
\hline TOTAL & 1074,81 & 0 & 26,37667997 & $-0,971779193$ & & & 58,84 \\
\hline
\end{tabular}

$$
\begin{gathered}
\lg a=\frac{26,37667997 \cdot 280}{14 \cdot 280}=1,884048569 \lg b=\frac{14 \cdot(-0,971779193)}{14 \cdot 280}=-0,003470639 \\
v_{\exp }=\left[\frac{\sum_{i=1}^{n}\left|\xi_{i}-\xi_{t_{i}}^{\exp }\right|}{n}: \frac{\sum_{i=1}^{n} \xi_{i}}{n}\right] \cdot 100=\frac{\sum_{i=1}^{n}\left|\xi_{i}-\xi_{t_{i}}^{\exp }\right|}{\sum_{i=1}^{n} \xi_{i}} \cdot 100=\frac{58,84}{1074,81} \cdot 100=5,47 \% \\
v_{I I}=4,12 \%<v_{\exp }=5,47 \%<v_{I}=5,57 \%
\end{gathered}
$$

The values regarding the Sony Corporation's worldwide revenues cover a quadratic „rally” $\xi_{t_{i}}=a+b \cdot t_{i}+c t_{i}^{2}$

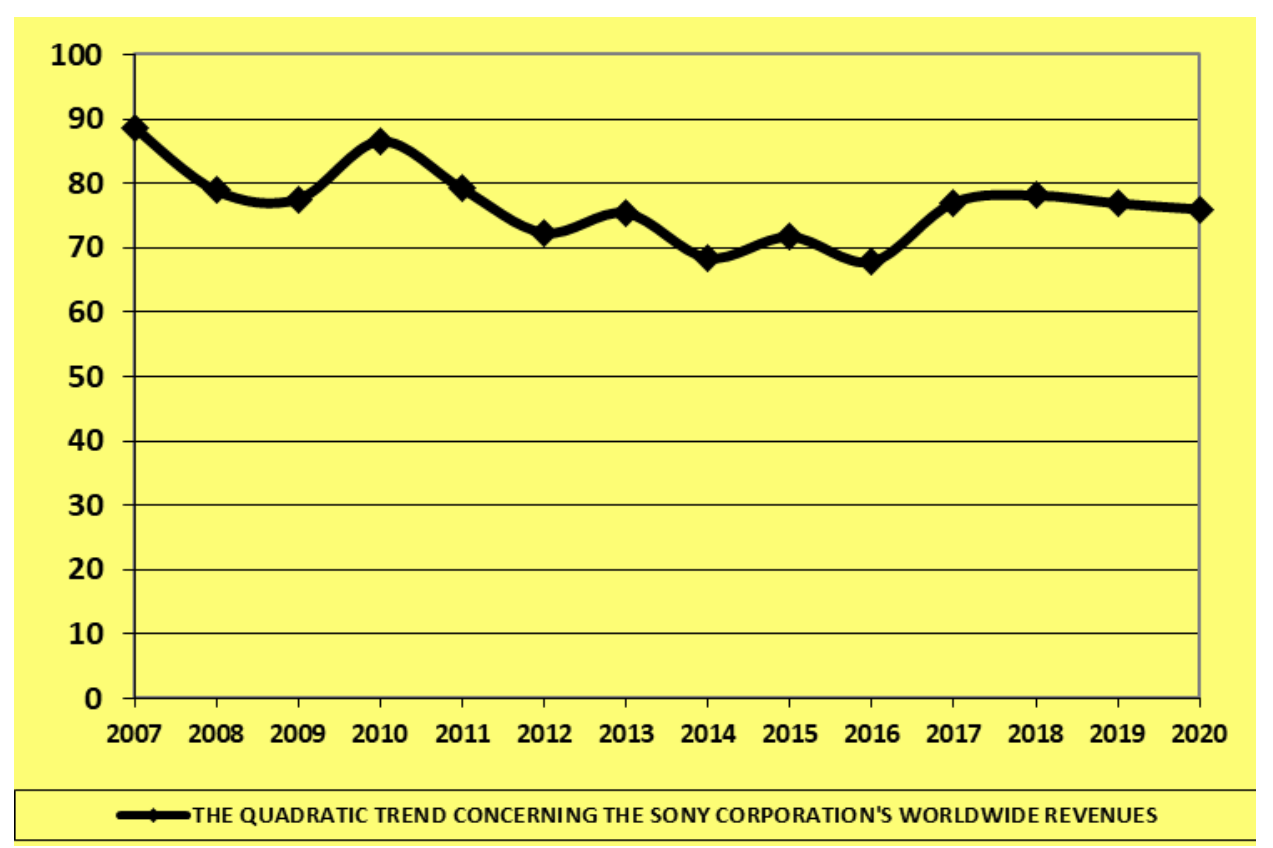

Graph 1. The quadratic „rally” for the values which show us

the evolution concerning the Sony Corporation's worldwide revenues

$$
\begin{aligned}
& \xi_{2021}^{\text {SONY'S_REVENUES }}=72,84607676+(-0,603392857) \cdot 8+0,196303304 \cdot 8^{2}=\$ 80,58 \text { _billions } \\
& \xi_{2022}^{\text {SONY'S_REVENUES }}=72,84607676+(-0,603392857) \cdot 9+0,196303304 \cdot 9^{2}=\$ 83,32 \text { _billions } \\
& \xi_{2023}^{\text {SONY'S_REVENUES }}=72,84607676+(-0,603392857) \cdot 10+0,196303304 \cdot 10^{2}=\$ 86,44 \text { _billions } \\
& \xi_{2024}^{\text {SONY'S_REVENUES }}=72,84607676+(-0,603392857) \cdot 11+0,196303304 \cdot 11^{2}=\$ 89,96 \text { _billions } \\
& \xi_{2025}^{\text {SONY'S_REVENUES }}=72,84607676+(-0,603392857) \cdot 12+0,196303304 \cdot 12^{2}=\$ 93,87 \text { _billions }
\end{aligned}
$$


3. The survey regarding the worldwide revenues for Sony Corporation regarding Imaging Product\&Solutions, which has as target them estimation between 2021-2025

Table 5. The Sony's Imaging Product\&Solutions worldwide revenues, between 2011-2019

\begin{tabular}{|c|c|}
\hline YEARS & $\begin{array}{c}\text { SONY'S IMAGING PRODUCTS\&SOLUTIONS } \\
\text { WORLDWIDE REVENUES } \\
\text { (billions\$) } \\
\left(\omega_{i}\right)\end{array}$ \\
\hline 2011 & $\mathbf{7 , 1 6}$ \\
\hline 2012 & $\mathbf{7 , 7 7}$ \\
\hline 2013 & $\mathbf{7 , 2 0}$ \\
\hline 2014 & $\mathbf{6 , 0 0}$ \\
\hline 2015 & $\mathbf{6 , 2 9}$ \\
\hline 2016 & $\mathbf{5 , 1 8}$ \\
\hline 2017 & $\mathbf{5 , 9 1}$ \\
\hline 2018 & $\mathbf{6 , 0 5}$ \\
\hline 2019 & $\mathbf{9 , 8 5}$ \\
\hline
\end{tabular}

- if the proceeding's structure, which draws up the trend for $\omega$ variable, where $\omega=$ the Sony's Imaging Product\&Solutions worldwide revenues, selects a linear exposure, $\omega_{t_{i}}=a+b \cdot t_{i}, a$ and $b$ will be [2]:

Table 6. The,esplanade” concerning the Sony's Imaging Product\&Solutions worldwide revenues, if these make a linear "rally"

\begin{tabular}{|c|c|c|c|c|c|c|}
\hline \multirow{2}{*}{ YEARS } & \multirow{2}{*}{$\begin{array}{c}\text { SONY'S IMAGING } \\
\text { PRODUCTS\&SOLUTIONS } \\
\text { WORLDWIDE REVENUES } \\
\text { (billions \$) } \\
\left(\omega_{i}\right)\end{array}$} & \multicolumn{5}{|c|}{ LINEAR TENDENCY } \\
\hline & & $t_{i}$ & $t_{i}^{2}$ & $t_{i} \omega_{i}$ & $\omega_{t_{i}}=a+b t_{i}$ & $\mid \omega_{i}-\omega_{t_{i}}$ \\
\hline 2011 & 7,16 & -4 & 16 & $-28,64$ & 6,676666666 & 0,48 \\
\hline 2012 & 7,77 & -3 & 9 & $-23,31$ & 6,713333333 & 1,06 \\
\hline 2013 & 7,20 & -2 & 4 & $-14,40$ & 6,750000000 & 0,45 \\
\hline 2014 & 6,00 & -1 & 1 & $-6,00$ & 6,786666666 & 0,79 \\
\hline 2015 & 6,29 & 0 & 0 & 0 & 6,823333333 & 0,53 \\
\hline 2016 & 5,18 & +1 & 1 & $+5,18$ & 6,860000000 & 1,68 \\
\hline 2017 & 5,91 & +2 & 4 & $+11,82$ & 6,896666666 & 0,99 \\
\hline 2018 & 6,05 & +3 & 9 & $+18,15$ & 6,933333333 & 0,88 \\
\hline 2019 & 9,85 & +4 & 16 & $+39,40$ & 6,970000000 & 2,88 \\
\hline TOTAL & 61,41 & 0 & 60 & 2,20 & & 9,74 \\
\hline
\end{tabular}

$$
\begin{gathered}
a=\frac{\sum_{i=1}^{n} \omega_{i} \sum_{i=1}^{n} t_{i}{ }^{2}-\sum_{i=1}^{n} \omega_{i} t_{i} \sum_{i=1}^{n} t_{i}}{n \sum_{i=1}^{n} t_{i}{ }^{2}-\left(\sum_{i=1}^{n} t_{i}\right)^{2}}=\frac{61,41 \cdot 60}{9 \cdot 60}=6,823333333 \\
b=\frac{n \sum_{i=1}^{n} \omega_{i} t_{i}-\sum_{i=1}^{n} t_{i} \sum_{i=1}^{n} \omega_{i}}{n \sum_{i=1}^{n} t_{i}{ }^{2}-\left(\sum_{i=1}^{n} t_{i}\right)^{2}}=\frac{9 \cdot 2,20}{9 \cdot 60}=0,036666666 \\
v_{I}=\left[\frac{\sum_{i=1}^{n}\left|\omega_{i}-\omega_{t_{i}}^{I}\right|}{n}: \frac{\sum_{i=1}^{n} \omega_{i}}{n}\right] \cdot 100=\frac{\sum_{i=1}^{n}\left|\omega_{i}-\omega_{t_{i}}^{I}\right|}{\sum_{i=1}^{n} \omega_{i}} \cdot 100=\frac{9,74}{61,41} \cdot 100=15,86 \%
\end{gathered}
$$

- if the proceeding's structure, which draws up the trend for $\omega$ variable, where $\omega=$ the Sony's Imaging Product\&Solutions worldwide revenues, selects a quadratic exposure, $\omega_{t_{i}}=a+b \cdot t_{i}+c t_{i}^{2}, a$ and $b$ will be [2]: 
Table 7. The „esplanade” regarding the Sony's Imaging Product\&Solutions worldwide revenues, if these make a quadratic "rally"

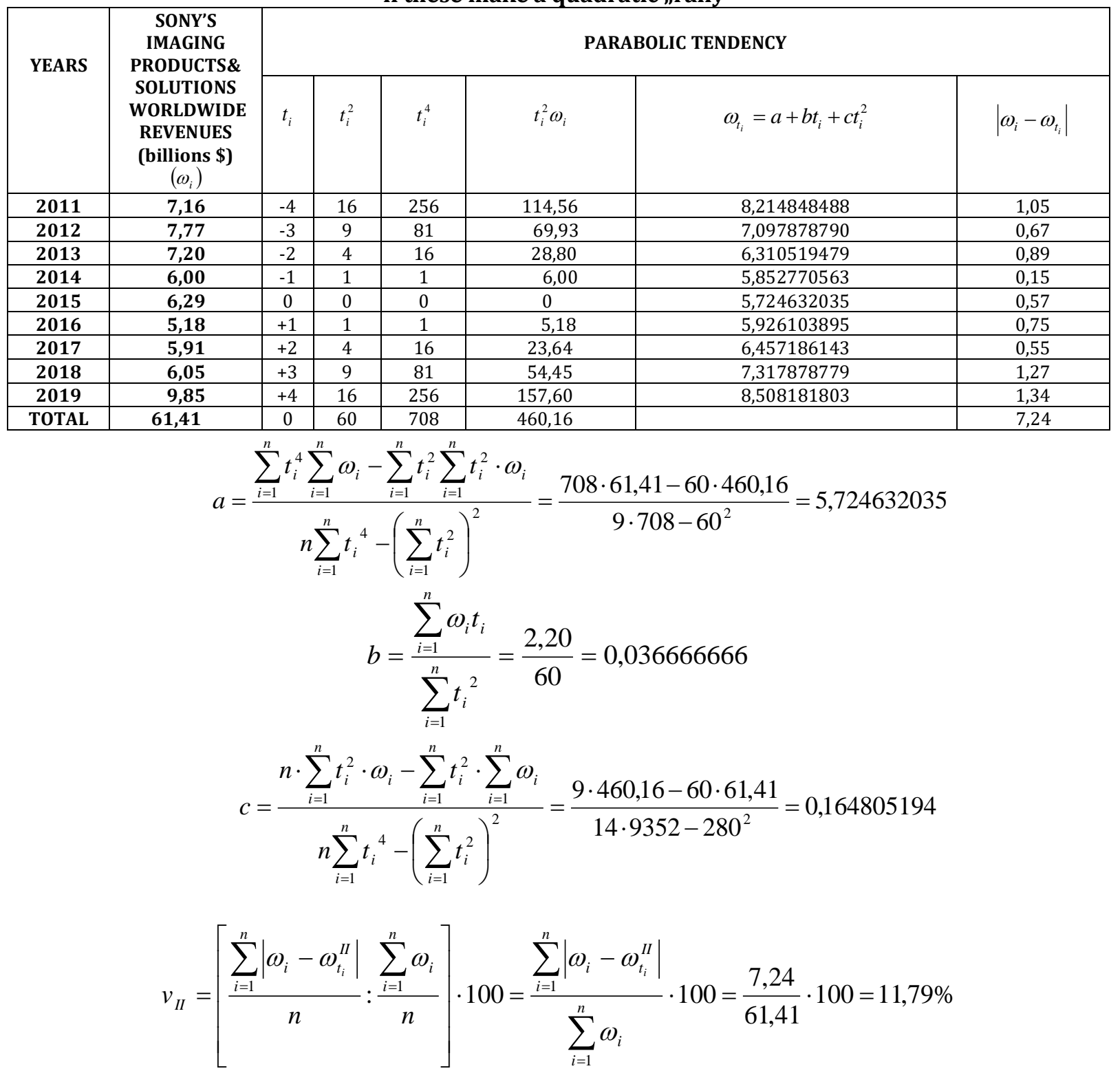

- if the proceeding's structure, which draws up the trend for $\omega$ variable, where $\omega=$ the Sony's Imaging Product\&Solutions worldwide revenues, selects an exponential exposure, $\omega_{t_{i}}=a b^{t_{i}}$, $a$ and $b$ will be [2]:

Table 8. The „esplanade” regarding the Sony's Imaging Product\&Solutions worldwide revenues, if these make an exponential "rally”

\begin{tabular}{|c|c|c|c|c|c|c|c|}
\hline \multirow{2}{*}{ YEARS } & \multirow{2}{*}{$\begin{array}{l}\text { SONY'S IMAGING } \\
\text { PRODUCTS\& } \\
\text { SOLUTIONS } \\
\text { WORLDWIDE } \\
\text { REVENUES } \\
\text { (billions \$) } \\
\left(\omega_{i}\right)\end{array}$} & \multicolumn{6}{|c|}{ EXPONENTIAL TENDENCY } \\
\hline & & $t_{i}$ & $\lg \omega_{i}$ & $t_{i} \lg \omega_{i}$ & $\lg \omega_{t_{i}}=\lg a+t_{i} \lg b$ & $\omega_{t_{i}}=a b^{t_{i}}$ & $\omega_{i}-\omega_{t_{i}}$ \\
\hline 2011 & 7,16 & -4 & 0,854913022 & $-3,419652089$ & 0,827211883 & 6,717565086 & 0,44 \\
\hline 2012 & 7,77 & -3 & 0,890421018 & $-2,671263056$ & 0,827091613 & 6,715705020 & 1,05 \\
\hline 2013 & 7,20 & -2 & 0,857332496 & $-1,714664993$ & 0,826971344 & 6,713845516 & 0,49 \\
\hline 2014 & 6,00 & -1 & 0,778151250 & $-0,778151250$ & 0,826851076 & 6,711986526 & 0,71 \\
\hline 2015 & 6,29 & 0 & 0,798650645 & 0 & 0,826730808 & 6,710128051 & 0,42 \\
\hline 2016 & 5,18 & +1 & 0,714329759 & $+0,714329759$ & 0,826610540 & 6,708270091 & 1,53 \\
\hline 2017 & 5,91 & +2 & 0,771587480 & $+1,543174962$ & 0,826490272 & 6,706412645 & 0,80 \\
\hline 2018 & 6,05 & +3 & 0,781755374 & $+2,345266124$ & 0,826370004 & 6,704555713 & 0,65 \\
\hline 2019 & 9,85 & +4 & 0,993436230 & $+3,973744922$ & 0,826249736 & 6,702699296 & 3,15 \\
\hline TOTAL & 61,41 & 0 & 7,440577275 & $-0,007216125$ & & & 9,24 \\
\hline
\end{tabular}




$$
\begin{gathered}
\lg a=\frac{\sum_{i=1}^{n} \lg \omega_{i} \sum_{i=1}^{n} t_{i}^{2}-\sum_{i=1}^{n} t_{i} \lg \omega_{i} \sum_{i=1}^{n} t_{i}}{n \sum_{i=1}^{n} t_{i}^{2}-\left(\sum_{i=1}^{n} t_{i}\right)^{2}}=\frac{7,440577275 \cdot 60}{9 \cdot 60}=0,826730808 \\
\lg b=\frac{n \cdot \sum_{i=1}^{n} t_{i} \lg \omega_{i}-\sum_{i=1}^{n} \lg \omega_{i} \sum_{i=1}^{n} t_{i}}{n \sum_{i=1}^{n} t_{i}^{2}-\left(\sum_{i=1}^{n} t_{i}\right)^{2}}=\frac{9 \cdot(-0,007216125)}{9 \cdot 60}=-0,000120268 \\
v_{\exp }=\left[\begin{array}{c}
\sum_{i=1}^{n}\left|\omega_{i}-\omega_{t_{i}}^{\exp }\right| \\
n
\end{array}: \frac{\sum_{i=1}^{n} \omega_{i}}{n}\right] \cdot 100=\frac{\sum_{i=1}^{n}\left|\omega_{i}-\omega_{t_{i}}^{\exp }\right|}{\sum_{i=1}^{n} \omega_{i}} \cdot 100=\frac{9,24}{61,41} \cdot 100=15,05 \% \\
v_{I I}=11,79 \%<v_{\text {exp }}=15,05 \%<v_{I}=15,86 \%
\end{gathered}
$$

The values concerning the Sony's Imaging Product\&Solutions worldwide revenues cover a quadratic „rally" $\omega_{t_{i}}=a+b \cdot t_{i}+c t_{i}^{2}$

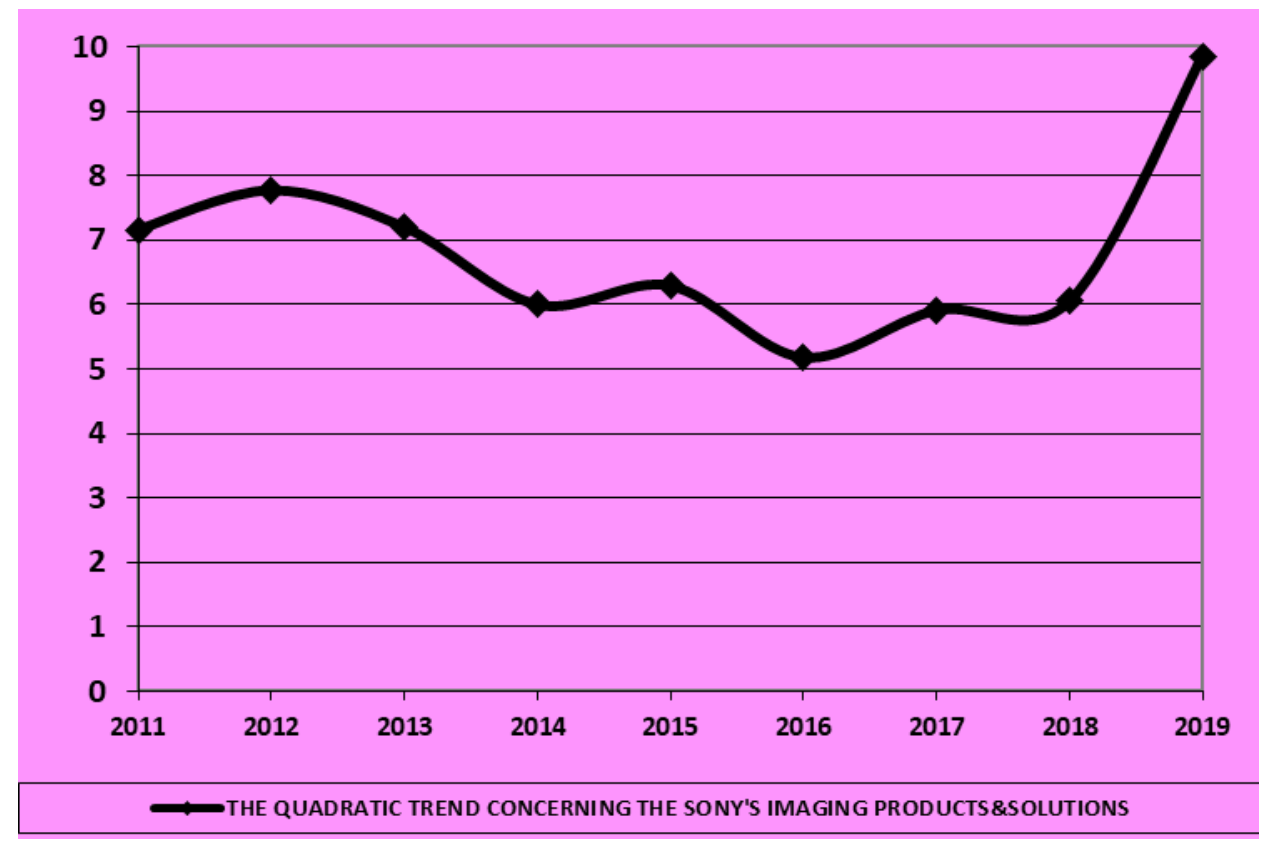

Graph 2. The quadratic „rally” for the values which show us the evolution regarding the Sony's Imaging Product\&Solutions worldwide revenues

\footnotetext{
$\omega_{2021}^{\text {SONY'S_IMAGING_PRODUCTS\&SOLUTIONS_REVENUES }}=5,724632035+0,036666666 \cdot 6+0,164805194 \cdot 6^{2}=\$ 11,88 \_$billions $\omega_{2022}^{\text {SONY'S_IMAGING_PRODUCTS\&SOLUTIONS_REVENUES }}=5,724632035+0,036666666 \cdot 7+0,164805194 \cdot 7^{2}=\$ 14,06$ _billions $\omega_{2023}^{\text {SONY'S_IMAGING_PRODUCTS\&SOLUTIONS _ REVENUES }}=5,724632035+0,036666666 \cdot 8+0,164805194 \cdot 8^{2}=\$ 16,57$ _billions $\omega_{2024}^{\text {SONY'S_IMAGING_PRODUCTS\&SOLUTIONS_REVENUES }}=5,724632035+0,036666666 \cdot 9+0,164805194 \cdot 9^{2}=\$ 19,40 \_$billions $\omega_{2025}^{\text {SONY'S_IMAGING_PRODUCTS\&SOLUTIONS_REVENUES }}=5,724632035+0,036666666 \cdot 10+0,164805194 \cdot 10^{2}=\$ 22,57 \_$billions
} 
4. The survey concerning the worldwide revenues for Sony's Home Entertainment and Sound Products\&Solutions, which has as target them estimation between 2021-2025

Table 9. The Sony's Home Entertainment and Sound Products\&Solutions worldwide revenues, between 2011-2018

\begin{tabular}{|c|c|}
\hline YEARS & $\begin{array}{c}\text { SONY'S HOME ENTERTAINMENT AND SOUND PRODUCTS\&SOLUTIONS } \\
\text { WORLDWIDE REVENUES } \\
\text { (billions\$) } \\
\left(\lambda_{i}\right)\end{array}$ \\
\hline 2011 & $\mathbf{1 2 , 0 6}$ \\
\hline 2012 & $\mathbf{1 0 , 5 8}$ \\
\hline 2013 & $\mathbf{1 1 , 3 5}$ \\
\hline 2014 & $\mathbf{1 0 , 0 6}$ \\
\hline 2015 & $\mathbf{1 0 , 2 6}$ \\
\hline 2016 & $\mathbf{9 , 2 8}$ \\
\hline 2018 & $\mathbf{1 1 , 0 3}$ \\
\hline & Source: „Statista Portal the United States of America”
\end{tabular}

- if the proceeding's structure, which draws up the trend for $\lambda$ variable, where $\lambda=$ the Sony's Home Entertainment and Sound Products\&Solutions worldwide revenues, selects a linear exposure, $\lambda_{t_{i}}=a+b \cdot t_{i}, a$ and $b$ will be [2]:

Table 10. The „esplanade” concerning the Sony's Home Entertainment and Sound Products\&Solutions worldwide revenues, if these make a linear „rally”

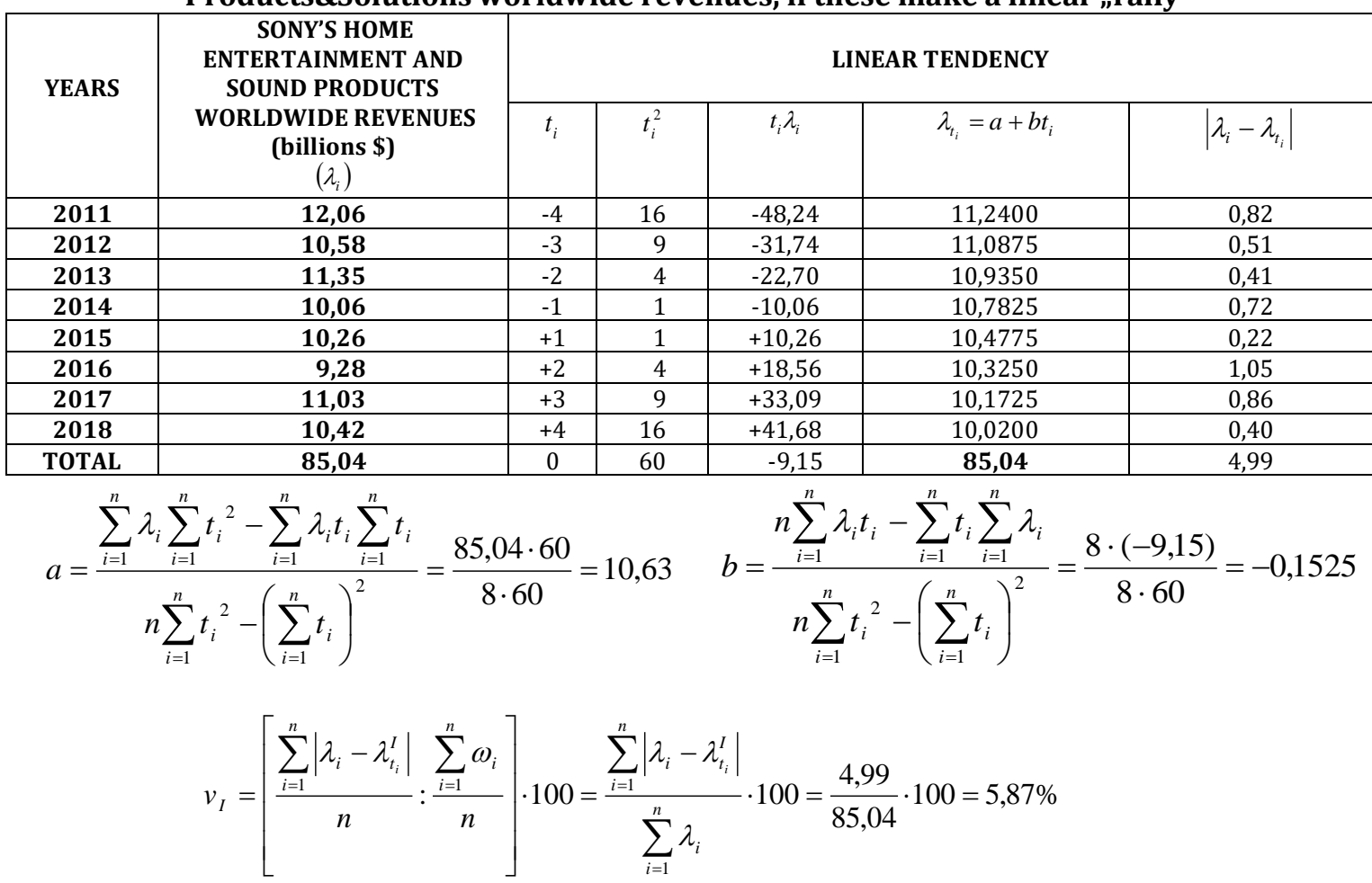

- if the proceeding's structure, which draws up the trend for $\lambda$ variable, where $\lambda=$ the Sony's Home Entertainment and Sound Products\&Solutions worldwide revenues, selects a quadratic exposure, $\lambda_{t_{i}}=a+b \cdot t_{i}+c t_{i}^{2}, a$ and $b$ will be [2]: 
Table 11. The,esplanade” regarding the Sony's Home Entertainment and Sound Products\&Solutions worldwide revenues, if these make a quadratic "rally”

\begin{tabular}{|c|c|c|c|c|c|c|c|}
\hline \multirow{2}{*}{ YEARS } & \multirow{2}{*}{$\begin{array}{c}\text { SONY'S HOME } \\
\text { ENTERTAINMENT } \\
\text { AND SOUND } \\
\text { PRODUCTS } \\
\text { WORLDWIDE } \\
\text { REVENUES } \\
\text { (billions \$) } \\
\left(\lambda_{i}\right)\end{array}$} & \multicolumn{6}{|c|}{ PARABOLIC TENDENCY } \\
\hline & & $t_{i}$ & $t_{i}^{2}$ & $t_{i}^{4}$ & $t_{i}^{2} \lambda_{i}$ & $\lambda_{t_{i}}=a+b t_{i}+c t_{i}^{2}$ & $\mid \lambda_{i}-\lambda_{t_{i}}$ \\
\hline 2011 & 12,06 & -4 & 16 & 256 & 192,96 & 11,87288759 & 0,19 \\
\hline 2012 & 10,58 & -3 & 9 & 81 & 95,22 & 11,19918605 & 0,62 \\
\hline 2013 & 11,35 & -2 & 4 & 16 & 45,40 & 10,67439923 & 0,68 \\
\hline 2014 & 10,06 & -1 & 1 & 1 & 10,06 & 10,29852713 & 0,24 \\
\hline 2015 & 10,26 & +1 & 1 & 1 & 10,26 & 9,993527134 & 0,27 \\
\hline 2016 & 9,28 & +2 & 4 & 16 & 37,12 & 10,06439923 & 0,78 \\
\hline 2017 & 11,03 & +3 & 9 & 81 & 99,27 & 10,28418605 & 0,75 \\
\hline 2018 & 10,42 & +4 & 16 & 256 & 166,72 & 10,65288759 & 0,23 \\
\hline TOTAL & 85,04 & 0 & 60 & 708 & 657,01 & & 3,76 \\
\hline
\end{tabular}

$$
a=\frac{\sum_{i=1}^{n} t_{i}^{4} \sum_{i=1}^{n} \lambda_{i}-\sum_{i=1}^{n} t_{i}{ }^{2} \sum_{i=1}^{n} t_{i}^{2} \cdot \lambda_{i}}{n \sum_{i=1}^{n} t_{i}{ }^{4}-\left(\sum_{i=1}^{n} t_{i}{ }^{2}\right)^{2}}=\frac{708 \cdot 85,04-60 \cdot 657,01}{8 \cdot 708-60^{2}}=10,07156977 \quad b=\frac{\sum_{i=1}^{n} \lambda_{i} t_{i}}{\sum_{i=1}^{n} t_{i}{ }^{2}}=-\frac{9,15}{60}=-0,1525
$$

$$
\begin{gathered}
c=\frac{n \cdot \sum_{i=1}^{n} t_{i}^{2} \cdot \lambda_{i}-\sum_{i=1}^{n} t_{i}^{2} \cdot \sum_{i=1}^{n} \lambda_{i}}{n \sum_{i=1}^{n} t_{i}^{4}-\left(\sum_{i=1}^{n} t_{i}^{2}\right)^{2}}=\frac{8 \cdot 657,01-60 \cdot 85,04}{8 \cdot 708-60^{2}}=0,074457364 \\
v_{I I}=\left[\frac{\sum_{i=1}^{n}\left|\lambda_{i}-\lambda_{t_{i}}^{I I}\right|}{n}: \frac{\sum_{i=1}^{n} \lambda_{i}}{n}\right] \cdot 100=\frac{\sum_{i=1}^{n}\left|\lambda_{i}-\lambda_{t_{i}}^{I I}\right|}{\sum_{i=1}^{n} \lambda_{i}} \cdot 100=\frac{3,764}{85,04} \cdot 100=4,42 \%
\end{gathered}
$$

- if the proceeding's structure, which draws up the trend for $\lambda$ variable, where $\lambda=$ the Sony's Home Entertainment and Sound Products\&Solutions worldwide revenues, selects an exponential exposure, $\lambda_{t_{i}}=a b^{t_{i}}, a$ and $b$ will be [2]:

Table 12 The „esplanade” regarding the Sony's Home Entertainment and Sound Products\&Solutions worldwide revenues, if these make an exponential „rally”

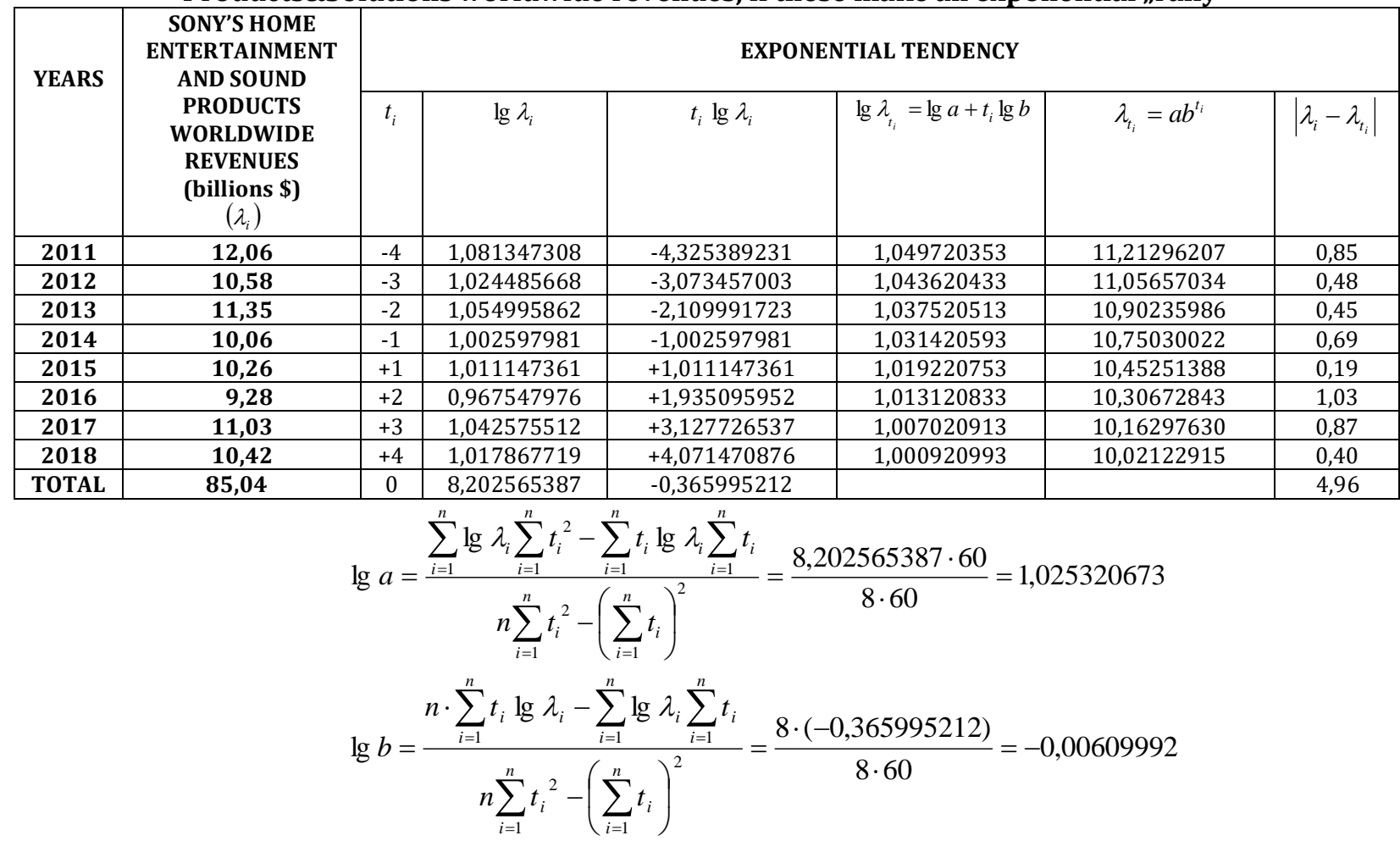




$$
v_{\exp }=\left[\frac{\sum_{i=1}^{n}\left|\lambda_{i}-\lambda_{t_{i}}^{\exp }\right|}{n}: \frac{\sum_{i=1}^{n} \lambda_{i}}{n}\right] \cdot 100=\frac{\sum_{i=1}^{n}\left|\lambda_{i}-\lambda_{t_{i}}^{\exp }\right|}{\sum_{i=1}^{n} \lambda_{i}} \cdot 100=\frac{4,96}{85,04} \cdot 100=5,83 \%
$$

The values concerning the Sony's Home Entertainment and Sound Products\&Solutions worldwide revenues cover a quadratic "rally” $\lambda_{t_{i}}=a+b \cdot t_{i}+c t_{i}^{2}$

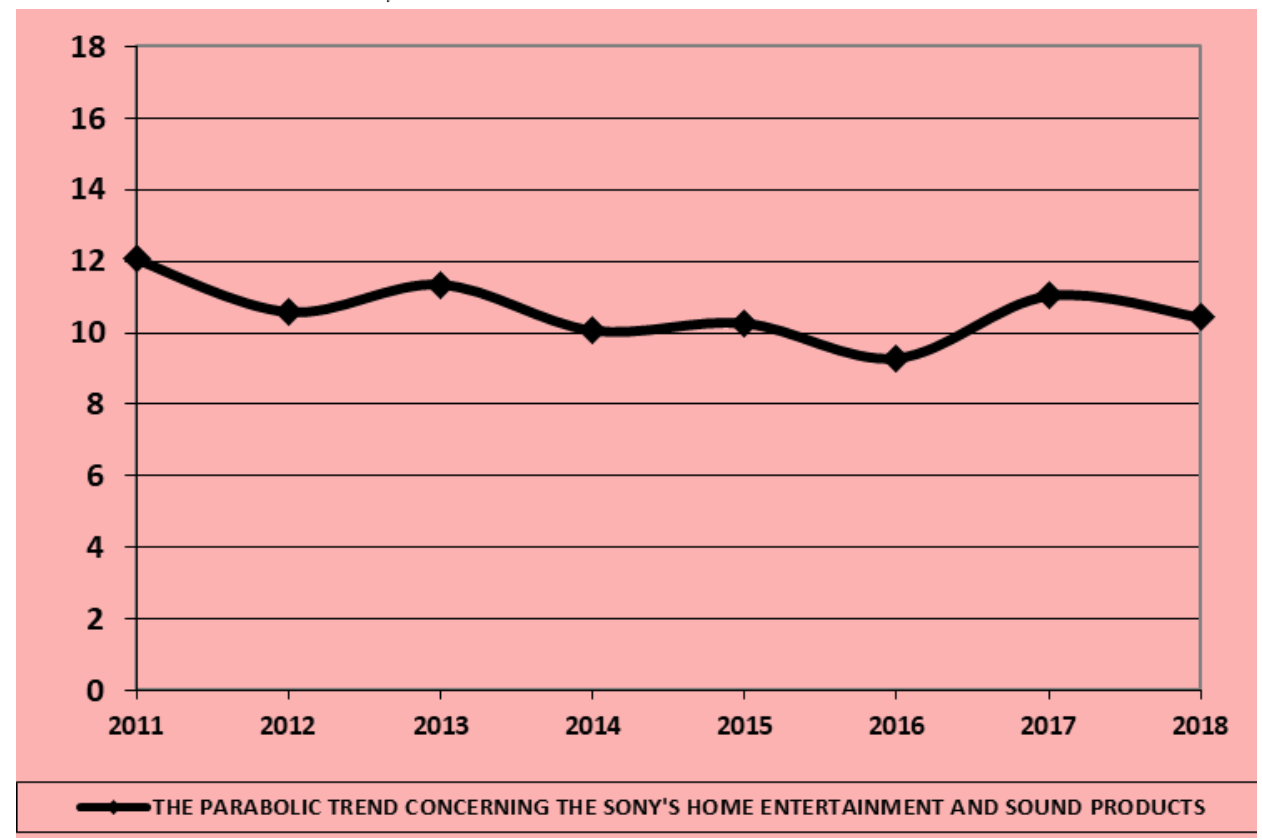

Graph 3. The quadratic „rally” for the values which show us the evolution for the Sony's Home Entertainment and Sound Products worldwide revenues

$\lambda_{2021}^{\text {SONY'S_HOME_ENTERTAINMENT \&SOUND_PRODUCTS\&SOLUTIONS_REVENUES }}=10,07156977+(-0,1525) \cdot 7+0,074457364 \cdot 7^{2}=\$ 12,65 \_$billions $\lambda_{2022}^{\text {SONY'S_HOME_ENTERTAINMENT \&SOUND_PRODUCTS\&SOLUTIONS_REVENUES }}=10,07156977+(-0,1525) \cdot 8+0,074457364 \cdot 8^{2}=\$ 13,62 \_$billions $\lambda_{2023}^{\text {SONY'S_HOME_ENTERTAINMENT\&SOUND_PRODUCTS\&SOLUTIONS_REVENUES }}=10,07156977+(-0,1525) \cdot 9+0,074457364 \cdot 9^{2}=\$ 14,73 \_$billions $\lambda_{2024}^{\text {SONY'S_HOME_ENTERTAINMENT \&SOUND_PRODUCTS\&SOLUTIONS_REVENUES }}=10,07156977+(-0,1525) \cdot 10+0,074457364 \cdot 10^{2}=\$ 15,99 \_$billions $\lambda_{2025}^{\text {SONY'S_HOME_ENTERTAINMENT\&SOUND_PRODUCTS\&SOLUTIONS_REVENUES }}=10,07156977+(-0,1525) \cdot 11+0,074457364 \cdot 11^{2}=\$ 17,40 \_$billions

5. The survey concerning the worldwide revenues for Sony Music Entertainment, which has as target them estimation between 2021-2025

Table 13. The Sony's Music Entertainment worldwide revenues, between 2011-2019

\begin{tabular}{|c|c|}
\hline YEARS & $\begin{array}{c}\text { SONY'S MUSIC ENTERTAINMENT } \\
\text { WORLDWIDE REVENUES } \\
\text { (billions \$) } \\
\left(\gamma_{i}\right)\end{array}$ \\
\hline 2011 & $\mathbf{4 , 1 6}$ \\
\hline 2012 & $\mathbf{4 , 7 0}$ \\
\hline $\mathbf{2 0 1 3}$ & $\mathbf{4 , 8 9}$ \\
\hline $\mathbf{2 0 1 4}$ & $\mathbf{4 , 5 4}$ \\
\hline $\mathbf{2 0 1 5}$ & $\mathbf{5 , 4 7}$ \\
\hline $\mathbf{2 0 1 6}$ & $\mathbf{5 , 7 8}$ \\
\hline $\mathbf{2 0 1 8}$ & $\mathbf{7 , 2 1}$ \\
\hline $\mathbf{2 0 1 9}$ & $\mathbf{7 , 2 8}$ \\
\hline & Source:, ,Statista Portal the United States of America” \\
\hline
\end{tabular}

- if the proceeding's structure, which draws up the trend for $\gamma$ variable, where $\gamma=$ the Sony's Music Entertainment worldwide revenues, selects a linear exposure, $\gamma_{t_{i}}=a+b \cdot t_{i}$, $a$ and $b$ will be [2]: 
Table 14. The „esplanade" concerning the Sony's Music Entertainment worldwide revenues, if these make a linear "rally"

\begin{tabular}{|c|c|c|c|c|c|c|}
\hline & \multirow{2}{*}{$\begin{array}{c}\text { SONY'S MUSIC } \\
\text { WORLDWIDE } \\
\text { YEARS }\end{array}$} & \multicolumn{6}{|c|}{$\begin{array}{l}\text { LINEAR TENDENCY } \\
\text { (billions \$) } \\
\end{array}$} & \begin{tabular}{c}
$\left.\gamma_{i}\right)$ \\
\cline { 3 - 7 }
\end{tabular} & $t_{i}$ & $t_{i}^{2}$ & $t_{i} \gamma_{i}$ & $\gamma_{t_{i}}=a+b t_{i}$ & $\left|\gamma_{i}-\gamma_{t_{i}}\right|$ \\
\hline $\mathbf{2 0 1 1}$ & $\mathbf{4 , 1 6}$ & -4 & 16 & $-16,64$ & 3,877111111 & 0,28 \\
\hline $\mathbf{2 0 1 2}$ & $\mathbf{4 , 7 0}$ & -3 & 9 & $-14,10$ & 4,348111111 & 0,35 \\
\hline $\mathbf{2 0 1 3}$ & $\mathbf{4 , 8 9}$ & -2 & 4 & $-9,78$ & 4,819111111 & 0,07 \\
\hline $\mathbf{2 0 1 4}$ & $\mathbf{4 , 5 4}$ & -1 & 1 & $-4,54$ & 5,290111111 & 0,75 \\
\hline $\mathbf{2 0 1 5}$ & $\mathbf{5 , 4 7}$ & 0 & 0 & 0 & 5,761111111 & 0,29 \\
\hline $\mathbf{2 0 1 6}$ & $\mathbf{5 , 7 8}$ & +1 & 1 & $+5,78$ & 6,232111111 & 0,45 \\
\hline $\mathbf{2 0 1 7}$ & $\mathbf{7 , 2 1}$ & +2 & 4 & $+14,42$ & 6,703111111 & 0,51 \\
\hline $\mathbf{2 0 1 8}$ & $\mathbf{7 , 2 8}$ & +3 & 9 & $+21,84$ & 7,174111111 & 0,11 \\
\hline $\mathbf{2 0 1 9}$ & $\mathbf{7 , 8 2}$ & +4 & 16 & $+31,28$ & 7,645111111 & 0,17 \\
\hline TOTAL & $\mathbf{5 1 , 8 5}$ & 0 & 60 & 28,26 & & 2,98 \\
\hline
\end{tabular}

$$
\begin{gathered}
a=\frac{\sum_{i=1}^{n} \gamma_{i} \sum_{i=1}^{n} t_{i}^{2}-\sum_{i=1}^{n} \gamma_{i} t_{i} \sum_{i=1}^{n} t_{i}}{n \sum_{i=1}^{n} t_{i}{ }^{2}-\left(\sum_{i=1}^{n} t_{i}\right)^{2}}=\frac{51,85 \cdot 60}{9 \cdot 60}=5,761111111 \quad b=\frac{n \sum_{i=1}^{n} \gamma_{i} t_{i}-\sum_{i=1}^{n} t_{i} \sum_{i=1}^{n} \gamma_{i}}{n \sum_{i=1}^{n} t_{i}{ }^{2}-\left(\sum_{i=1}^{n} t_{i}\right)^{2}}=\frac{9 \cdot 28,26}{9 \cdot 60}=0,471 \\
v_{I}=\left[\frac{\sum_{i=1}^{n}\left|\gamma_{i}-\gamma_{t_{i}}^{I}\right|}{n}: \frac{\sum_{i=1}^{n} \gamma_{i}}{n}\right] \cdot 100=\frac{\sum_{i=1}^{n}\left|\gamma_{i}-\gamma_{t_{i}}^{I}\right|}{\sum_{i=1}^{n} \gamma_{i}} \cdot 100=\frac{2,98}{51,85} \cdot 100=5,75 \%
\end{gathered}
$$

- if the proceeding's structure, which draws up the trend for $\gamma$ variable, where $\gamma=$ the Sony's Music

Entertainment worldwide revenues, selects a quadratic exposure, $\gamma_{t_{i}}=a+b \cdot t_{i}+c t_{i}^{2}, a$ and $b$ will be [2]:

Table 15. The,esplanade” regarding the Sony's Music Entertainment worldwide revenues,

\begin{tabular}{|c|c|c|c|c|c|c|c|}
\hline \multirow{2}{*}{ YEARS } & \multirow{2}{*}{$\begin{array}{c}\text { SONY'S MUSIC } \\
\text { WORLDWIDE } \\
\text { REVENUES } \\
\text { (billions \$) } \\
\left(\gamma_{i}\right)\end{array}$} & \multicolumn{6}{|c|}{ PARABOLIC TENDENCY } \\
\hline & & $t_{i}$ & $t_{i}^{2}$ & $t_{i}^{4}$ & $t_{i}^{2} \gamma_{i}$ & $\gamma_{t_{i}}=a+b t_{i}+c t_{i}^{2}$ & $\left|\gamma_{i}-\gamma_{t_{i}}\right|$ \\
\hline 2011 & 4,16 & -4 & 16 & 256 & 66,56 & 4,257515151 & 0,10 \\
\hline 2012 & 4,70 & -3 & 9 & 81 & 42,30 & 4.443212114 & 0,26 \\
\hline 2013 & 4,89 & -2 & 4 & 16 & 19,56 & 4,710424239 & 0,18 \\
\hline 2014 & 4,54 & -1 & 1 & 1 & 4,54 & 5,059151514 & 0,52 \\
\hline 2015 & 5,47 & 0 & 0 & 0 & 0 & 5,489393939 & 0,02 \\
\hline 2016 & 5,78 & +1 & 1 & 1 & 5,78 & 6,001151514 & 0,22 \\
\hline 2017 & 7,21 & +2 & 4 & 16 & 28,84 & 6,594424239 & 0,62 \\
\hline 2018 & 7,28 & +3 & 9 & 81 & 65,52 & 7,269212114 & 0,01 \\
\hline 2019 & 7,82 & +4 & 16 & 256 & 125,12 & 8,025515139 & 0,21 \\
\hline TOTAL & 51,85 & 0 & 60 & 708 & 358,22 & & 2,14 \\
\hline
\end{tabular}
if these make a quadratic „rally”

$$
\begin{gathered}
a=\frac{\sum_{i=1}^{n} t_{i}^{4} \sum_{i=1}^{n} \gamma_{i}-\sum_{i=1}^{n} t_{i}^{2} \sum_{i=1}^{n} t_{i}^{2} \cdot \gamma_{i}}{n \sum_{i=1}^{n} t_{i}^{4}-\left(\sum_{i=1}^{n} t_{i}^{2}\right)^{2}}=\frac{708 \cdot 51,85-60 \cdot 358,22}{9 \cdot 708-60^{2}}=5,489393939 \quad b=\frac{\sum_{i=1}^{n} \gamma_{i} t_{i}}{\sum_{i=1}^{n} t_{i}^{2}}=\frac{28,26}{60}=0,471 \\
c=\frac{n \cdot \sum_{i=1}^{n} t_{i}^{2} \cdot \gamma_{i}-\sum_{i=1}^{n} t_{i}^{2} \cdot \sum_{i=1}^{n} \gamma_{i}}{n \sum_{i=1}^{n} t_{i}^{4}-\left(\sum_{i=1}^{n} t_{i}^{2}\right)^{2}}=\frac{9 \cdot 358,22-60 \cdot 51,85}{9 \cdot 708-60^{2}}=0,040757575 \\
v_{I I}=\left[\frac{\sum_{i=1}^{n}\left|\gamma_{i}-\gamma_{t_{i}}^{I I}\right|}{n}: \frac{\sum_{i=1}^{n} \gamma_{i}}{n}\right] \cdot 100=\frac{\sum_{i=1}^{n}\left|\gamma_{i}-\gamma_{t_{i}}^{I I}\right|}{\sum_{i=1}^{n} \gamma_{i}} \cdot 100=\frac{2,14}{51,85} \cdot 100=4,13 \%
\end{gathered}
$$


- if the proceeding's structure, which draws up the trend for $\gamma$ variable, where $\gamma=$ the Sony's Music

Entertainment worldwide revenues, selects an exponential exposure, $\gamma_{t_{i}}=a b^{t_{i}}, a$ and $b$ will be [2]:

Table 16. The „esplanade” regarding the Sony's Music Entertainment worldwide revenues, if these make an exponential "rally"

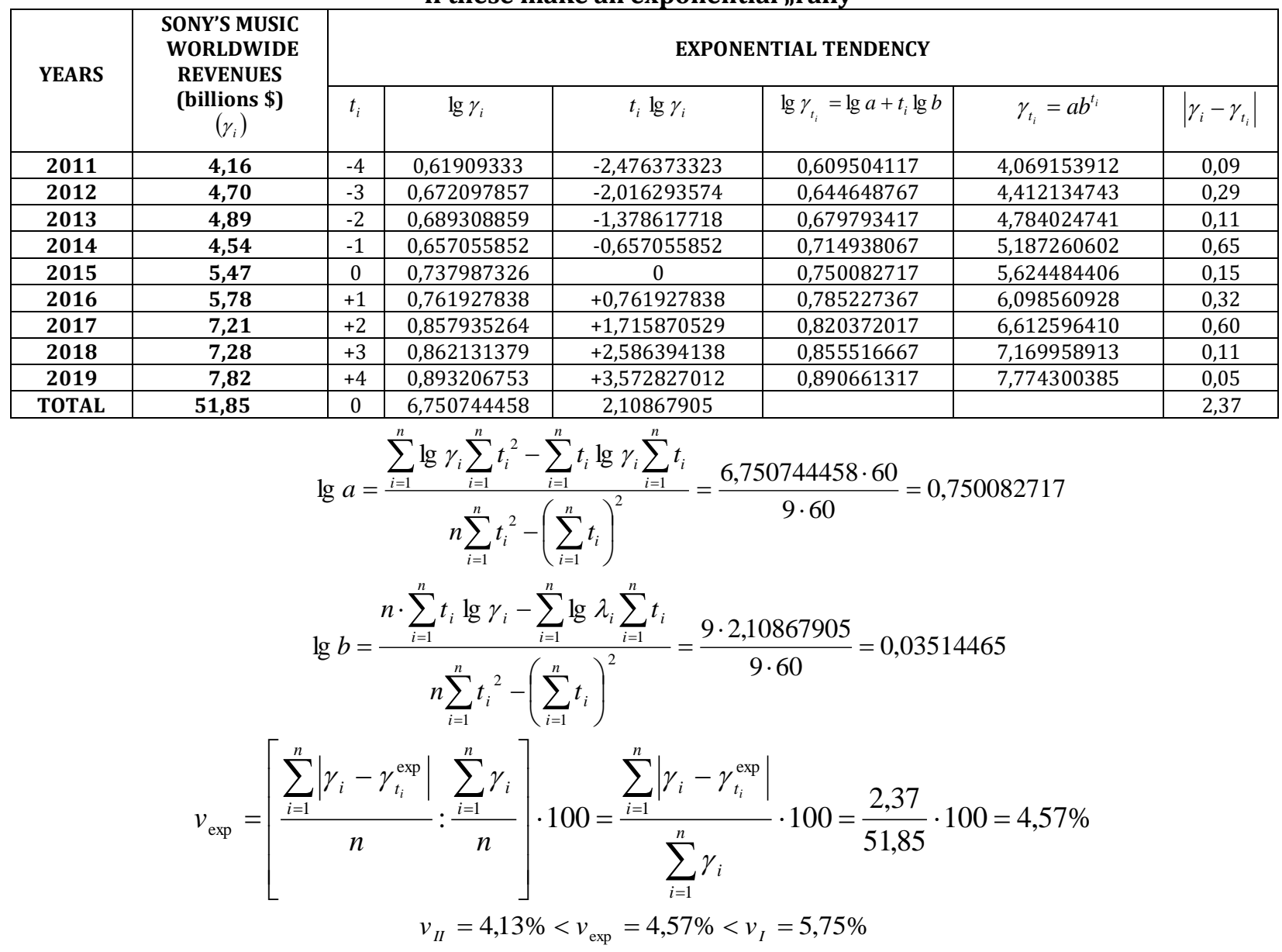

The values concerning the Sony's Music Entertainment worldwide revenues cover a quadratic "rally” $\gamma_{t_{i}}=a+b \cdot t_{i}+c t_{i}^{2}$

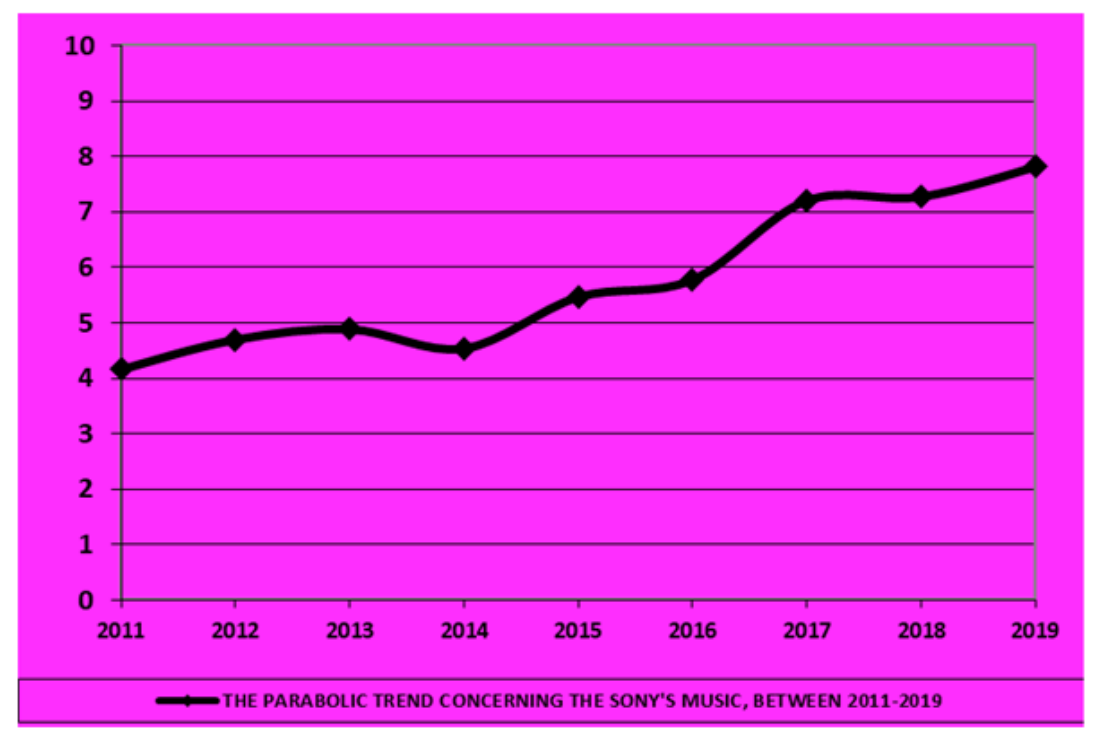

Graph 4. The quadratic „rally” for the values which show us the evolution concerning the Sony's Music Entertainment worldwide revenues

$\gamma_{2021}^{\text {SONY'S_MUSIC_REVENUES }}=5,489393939+0,471 \cdot 6+0,040757575 \cdot 6^{2}=\$ 9,78 \_$billions 


$$
\begin{aligned}
& \gamma_{2022}^{\text {SONY'S_MUSIC_REVENUES }}=5,489393939+0,471 \cdot 7+0,040757575 \cdot 7^{2}=\$ 10,78 \_ \text {billions } \\
& \gamma_{2023}^{\text {SONY'S_MUSIC_REVENUES }}=5,489393939+0,471 \cdot 8+0,040757575 \cdot 8^{2}=\$ 11,87 \text { _billions } \\
& \gamma_{2024}^{\text {SONY'S_MUSIC_REVENUES }}=5,489393939+0,471 \cdot 9+0,040757575 \cdot 9^{2}=\$ 13,03 \text { _billions } \\
& \gamma_{2025}^{\text {SONY'S_MUSIC_REVENUES }}=5,489393939+0,471 \cdot 10+0,040757575 \cdot 10^{2}=\$ 14,27 \text { billions }
\end{aligned}
$$

\section{Conclusions}

In Forbes, the Sony Corporation has the positioning on 47 place, in the list named „the 2020 world's most valuable brands", with \$13,3 billions quotation for the brand value. We can remark between 20212025 , that the Sony Corporation's worldwide revenues will be in a continuous augmentation, from $\$ 80,58$ billions, to \$93,87 billions. Also, the Sony's Imaging Product\&Solutions worldwide revenues will manifest the same „symptom”, namely permanent increment in the period 2021-2025, from $\$ 11,88$ billions, to $\$ 22,57$ billions, concerning the digital imaging products and the medical goods. Concerning the Sony's Home Entertainment and Sound Products\&Solutions worldwide revenues, there are rises in future, between 20212025 , from $\$ 12,65$ billions, to $\$ 17,40$ billions. Alike, the Sony's Music worldwide revenues will be in a continuous development between 2021-2025, from $\$ 9,78$ billions, to $\$ 14,27$ billions.

\section{References}

[1]. Asakura R. (2000). Revolutionaries at Sony: The making of the Sony Playstation and the Visionaries who Conquered the World of Video Games, McGraw-Hill Publishing House, New-York.

[2]. Gauss C.F. (1986). Disquisitiones Arithmeticae and other papers on number theory, english translation Springer Publishing House, NewYork, 1986.

[3]. Morita A., Reingold E., Shimomura M. (1986). Made in Japan: Akio Morita and Sony, Dutton Books Publishing House, New-York, 1986.

[4]. Sudjic D. (2015). Sony Design: Making Modern, Rizzoli Publishing House, Milan, 2015.

[5]. Wood D. (2012). Sony Vegas Pro 11 Beginner's Guide, Packt Publishing House, Birmingham, 2012. 